\title{
Increasing dominant follicular proportion negatively associated with good clinical outcomes in GnRH-a prolonged protocol: a large-sample retrospective analysis
}

\section{Houming Su}

Tongji Hospital of Tongji Medical College of Huazhong University of Science and Technology

\section{Youhua Lai}

Tongji Hospital of Tongji Medical College of Huazhong University of Science and Technology Jie Li

Tongji Hospital of Tongji Medical College of Huazhong University of Science and Technology Tingting Liao

Tongji Hospital of Tongji Medical College of Huazhong University of Science and Technology

\section{Licheng Ji}

Tongji Hospital of Tongji Medical College of Huazhong University of Science and Technology

\section{Xinyao Hu}

Tongji Hospital of Tongji Medical College of Huazhong University of Science and Technology

Kun Qian ( $\square$ kunqian1129@yeah.net)

Department of Rehabilitation Medicine, Tongji Hospital, Tongji Medical College, Huazhong University of Science and Technology https://orcid.org/0000-0001-8063-457X

\section{Research}

Keywords: IVF/ICSI, controlled ovarian hyperstimulation, HCG trigger time, GnRH-a prolonged protocol, dominant follicular proportion, clinical outcomes

Posted Date: September 7th, 2021

DOl: https://doi.org/10.21203/rs.3.rs-870140/v1

License: (c) (i) This work is licensed under a Creative Commons Attribution 4.0 International License. Read Full License 


\section{Increasing dominant follicular proportion negatively associated with good clinical outcomes in GnRH-a prolonged protocol: a large-sample retrospective analysis}

1 Houming Su, Youhua Lai, Jie Li, Tingting Liao, Licheng Ji, Xinyao Hu, Kun Qian*

2 Reproductive Medicine Center, Tongji Hospital, Tongji Medical College, Huazhong University

3 of Science and Technology, Wuhan, China

$4 *$ Correspondence:

5 Kun Qian

6 kunqian@tjh.tjmu.edu.cn

\section{Abstract}

8 Background: Nowadays, there is no universal criteria for trigger time during controlled ovarian

9 hyperstimulation (COH). Particularly, in the so-called GnRH-a prolonged protocol, widely used in

10 China, the ideal time to trigger ovulation is not yet well defined.

11 Methods: This was a large-sample retrospective analysis. Between January 2016 and January 2020,

121,925 young patients who underwent their first in vitro fertilization (IVF)/intracytoplasmic sperm

13 injection (ICSI) cycles with normal ovarian response were divided into three groups based on their

14 dominant follicular proportions (DFP, defined as $\geqslant 18 \mathrm{~mm}$ follicles $/ \geqslant 14 \mathrm{~mm}$ follicles; Group A: <

$1530 \%$; Group B: 30\% - 60\%; Group C: $\geqslant 60 \%$ ). Binary logistic regression and multivariate linear

16 regression were used to assessed whether DFP levels were related to clinical pregnancy, number of

17 blastocysts frozen, blastocyst formation rate and low blastocysts frozen. 
18 Results: Binary logistics regression analysis showed that compared with Group A, the OR for

19 clinical pregnancy was 1.345 in Group B $(\mathrm{P}=0.023)$; however, there was no statistical difference

20 between Group C and Group A $(\mathrm{P}=0.216)$. On one hand, multivariate linear regression analysis

21 indicated that DFP was negatively associated with number of blastocysts frozen $(\beta \pm \mathrm{SE}$ : Group B vs

22 Group $\mathrm{A}=-0.319 \pm 0.115, \mathrm{P}=0.006$; Group $\mathrm{C}$ vs Group $\mathrm{A}=-0.432 \pm 0.154, \mathrm{P}=0.005)$ as well as

23 blastocyst formation rate $(\beta \pm \mathrm{SE}$ : Group B vs Group $\mathrm{A}=-0.035 \pm 0.016, \mathrm{P}=0.031$; Group $\mathrm{C}$ vs

24 Group $\mathrm{A}=-0.039 \pm 0.021, \mathrm{P}=0.067)$. On the other hand, compared with group $\mathrm{A}$, the OR for low

25 blastocyst frozen was 1.312 in Group $\mathrm{B}(\mathrm{P}=0.039)$ and was 1.417 in Group $\mathrm{C}(\mathrm{P}=0.041)$.

26 Conclusions: Excessive delay of trigger in GnRH-a prolonged protocol might reduce the

27 developmental potential of oocytes and reduce the number of available blastocysts, which might

28 result in a lower cumulative pregnancy rate. But further confirmation by strict prospective

29 randomized controlled study should be needed.

30 Trial registration: https://clinicaltrials.gov/; NCT03305510; Registered 08 October 2017 -

31 Retrospectively registered.

32 Keywords: IVF/ICSI, controlled ovarian hyperstimulation, HCG trigger time, GnRH-a prolonged

33 protocol, dominant follicular proportion, clinical outcomes.

\section{Background}

$35 \mathrm{COH}$ is considered a key factor in the success of IVF/ICSI [1, 2], because it induces the development

36 of multiple follicles and obtains as many high-quality oocytes as possible, thereby increasing the

37 numbers of available embryos for transfer and increasing pregnancy rates. A crucial step in

38 improving clinical pregnancy outcomes, however, is identifying an appropriate time for human

39 chorionic gonadotropin (HCG) trigger [2, 3]. 
40 To date, there has been no universal criteria for the optimal HCG trigger time for IVF/ICSI cycles in

41 a variety of $\mathrm{COH}$ protocols $[1,2,5]$. Traditionally, the majority of reproductive centers administer

42 the trigger when at least three leading follicles have reached $\geqslant 17 \mathrm{~mm}$, or at least two leading

43 follicles have reached $\geqslant 18 \mathrm{~mm}[1,2,6-8]$. Ectors et al. proposed to postpone HCG trigger in short

44 GnRH-a protocol. They suggested that it may yield relatively more large follicles, including

45 competent oocytes with mature cytoplasm, and may exert positive effects on clinical outcomes [9].

46 On the contrary, some studies suggested that delaying oocyte retrieval in short GnRH-a long protocol

47 increased neither the number of mature oocytes retrieved nor the pregnancy rate and may, in fact,

48 impair embryo quality [8]. However, other studies did not find differences in fertilization rate or

49 oocyte maturation rate between large and small follicles (10 - $14 \mathrm{~mm} ; 15-19 \mathrm{~mm} ; 20+\mathrm{mm})$ [10];

$50 \quad(<16 \mathrm{~mm} ; \geqslant 16 \mathrm{~mm})[11]$. These discordant findings might be attributed to differences in $\mathrm{COH}$

51 protocols, definition of follicle size, patient characteristics, methods of oocyte insemination (IVF or

52 ICSI), and analysis of endpoints [1].

53 In recent years, IVF and ICSI have developed rapidly, and the available COH protocols are flexible

54 and diverse. For example, the GnRH-a prolonged protocol is an emerging protocol that is widely

55 used in clinical practice (especially in China). Nevertheless, the ideal time to trigger ovulation has not

56 yet been well defined for this protocol. Some reproductive centers simply follow the criteria of

57 trigger time of short GnRH-a long protocol when using this protocol. However, the mechanism of

58 action, endocrine hormones, and medication regimen differ greatly between different $\mathrm{COH}$ protocols

59 [4]. It's controversial to choose the same trigger time for different $\mathrm{COH}$ protocols [1, 19]. Evidence is

60 still urgently needed to provide a reference for clinical decision-making regarding the trigger time of

61 GnRH-a prolonged protocol. 
62 Folliculogenesis may be asynchronous during ovarian hyperstimulation. We need to focus on the

63 global development state of the growing follicle group [8]. The dominant follicular proportion (DFP)

64 was first proposed in the GnRH antagonist protocol, which can reflect the overall development of

65 follicles. And it was considered to be a more effective and objective indicator to determine the ideal

66 HCG trigger timing [5]. In this study, we compared the effects of different DFP ( $\geqslant 18 \mathrm{~mm}$ follicles /

$67 \geqslant 14 \mathrm{~mm}$ follicles) on clinical outcomes in a large group of people who underwent IVF or ICSI

68 treatment with GnRH-a prolonged protocol, including oocyte maturation rate, normal fertilization

69 rate, clinical pregnancy rate, implantation rate, number of blastocysts frozen, blastocyst formation

70 rate, and low blastocyst frozen.

\section{Methods}

\section{$72 \quad$ Patient selection}

73 This is a retrospective study. All eligible patients who underwent and completed their first IVF/ICSI

74 cycle with GnRH-a prolonged protocol from January 2016 to January 2020 at the Reproductive

75 Medicine Center of Tongji Hospital, Affiliated to Huazhong University of Science and Technology,

76 were recruited and analyzed retrospectively.

77 The inclusion criteria were as follows: (1) the first IVF/ICSI fresh embryo transfer cycle, (2) female

78 with age $<35$ years, (3) body mass index (BMI) $\leqslant 30 \mathrm{~kg} / \mathrm{m} 2$, (4) antral follicle counting (AFC) $\geqslant 5$,

79 (5) anti-Müllerian hormone (AMH) $\geqslant 1.2 \mathrm{IU} / \mathrm{L}$, (6) basal serum follicle-stimulating hormone (FSH)

$80<12$ IU/L, and (7) $6-18$ retrieved oocytes.

81 The exclusion criteria were as follows: (1) donor oocyte cycles/oocyte cryopreservation cycles, (2)

82 female who had previously undergone an IVF/ICSI cycle either in our center or elsewhere. 


\section{Controlled ovarian stimulation protocols}

84 For GnRH-a prolonged protocol, $3.75 \mathrm{mg}$ of long-acting GnRH-a (Decapeptyl; Ferring, Saint-Prex,

85 Switzerland) was administered subcutaneously on day 2 of the menstrual cycle. Pituitary suppression

86 was evaluated 28 days after pituitary downregulation. The criteria for confirming the success of

87 downregulation were as follows: follicle diameter $<5 \mathrm{~mm}$, serum luteinizing hormone (LH) $<5$

$88 \mathrm{mIU} / \mathrm{ml}$, serum estradiol (E2) $<50 \mathrm{pg} / \mathrm{mL}$, and endometrial thickness $<5 \mathrm{~mm}$. Then, daily injection

89 of rhFSH (Gonal-F; Merck-Serono, Geneva, Switzerland) ranging from 75 to 300 IU was given for

90 about 10 days. When at least three leading follicles $\geqslant 17 \mathrm{~mm}$ or two leading follicles $\geqslant 18 \mathrm{~mm}$ were

91 observed via transvaginal ultrasound, a dose of 0.25mg rHCG (Ovidrel; Merck-Serono, Geneva,

92 Switzerland) was administered to trigger ovulation. After 36 - 37 hours, transvaginal ultrasound-

93 guided oocyte retrieval was conducted. Fertilization was accomplished by standard IVF or ICSI. If

94 serum progesterone $<1.5 \mathrm{ng} / \mathrm{mL}$ [17], the number of oocytes retrieved were <20, and serum E2 were

$95<7,000 \mathrm{pg} / \mathrm{mL}$, the one or two best-quality Day 3 embryos were transferred; and the remaining

96 embryos were cultured to Day 5/6 until the blastocyst formed and then frozen. The luteal phase was

97 supported if embryo transfer was performed.

98 Definition of DFP Levels and Groups

99 Dominant follicular proportion (DFP) is defined as $\geqslant 18 \mathrm{~mm}$ follicles $/ \geqslant 14 \mathrm{~mm}$ follicles. DFP $<$

$10030 \%$ corresponds to at least two follicles $\geqslant 18 \mathrm{~mm}$ on HCG day, which is the most common trigger

101 time. Then, we set the groups as DFP $<30 \%$ (Group A), 30\% - 60\% (Group B), $\geqslant 60 \%$ (Group C)

102 corresponding to follicles $\geqslant 18 \mathrm{~mm}$ increasing gradually $(2,4,6$ follicles respectively).

\section{Clinical outcomes}


104 The number of mature oocytes (MII) was measured 3 - 4 hours, and fertilization was assessed 16 - 18

105 hours after IVF insemination or when ICSI was performed. Oocyte maturation rate was the

106 proportion of MII oocytes to the number of oocytes retrieved. Normal fertilization rate was equal to

107 2PN/number of oocytes retrieved (IVF) or 2PN/MII (ICSI). The blastocyst formation rate was equal

108 to the number of blastocysts frozen divided by the number of blastocysts continuously cultured. Low

109 blastocysts frozen was defined as the number of blastocysts frozen $\leqslant 1$ (that is, less than half of the

110 average number of blastocysts frozen). Clinical pregnancy was identified with the presence of an

111 intrauterine gestational sac with fetal cardiac activity. The clinical pregnancy rate was the number of

112 clinical pregnancies in a given number of transplant cycles. Implantation rate reflects the number of

113 gestational sacs divided by the number of embryos transferred.

\section{Statistical analysis}

115 Pearson chi-square $(\chi 2)$ test on categorical variables and analysis of variance (ANOVA) or Kruskall-

116 Wallis $\mathrm{H}$ test on continuous variables were performed appropriately. Multivariate linear regression

117 analyses were carried out for the predictive factors of number of blastocysts frozen and blastocyst

118 formation rate. Moreover, binary logistic regression analyze was carried out for the predictive factors

119 of clinical pregnancy and low blastocyst frozen. The results were given in terms of $95 \%$ confidence

120 intervals (CI) and P values. A two tailed P-value of $<0.05$ indicated statistical significance. All

121 statistical analyses of the data were carried out using the Statistical Package for Social Science 122 version 25.0 (SPSS, Chicago, IL, USA).

\section{Results}

124 A total of 1,925 consecutive IVF / ICSI cycles were included. As shown in Table 1, the clinical

125 characteristics, ovarian response characteristics, and reproductive outcomes of cycles were described.

126 The baseline characteristics and ovarian stimulation details were matched evenly in three groups, 
127 including maternal age, BMI, basal FSH, dose of Gn, duration of Gn. Among the DFP groups, there

128 were no significant differences in terms of oocyte maturation rate (IVF/ICSI), normal fertilization

129 rate (IVF/ICSI), and clinical pregnancy rate. Group B had the highest implantation rate (Group A

$13048.67 \%$, Group B 57.57\%, Group C 50.71\%, P = 0.031). Interestingly, Group A had the highest

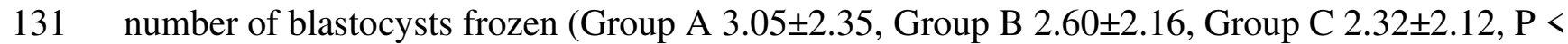

1320.001 ) and the highest blastocyst formation rate (Group A 42.38\% $\pm 26.59 \%$, Group B 38.69\% \pm

$13327.16 \%$, Group C $38.26 \% \pm 28.27 \%, \mathrm{P}=0.047)$.

134 Binary logistic regression analysis was carried out to evaluate the effect of BMI, dose/duration of Gn,

135 number of oocytes retrieved, number of embryo transfer and DFP levels on clinical pregnancy

136 (Table 2). The results showed that compared with Group A, the OR for clinical pregnancy was 1.345

137 in Group B (P = 0.023); however, there was no statistical difference between Group C and Group A

$138 \quad(\mathrm{P}=0.216)$

139 Multivariate linear regression analyses were carried out to evaluate the effect of type of infertility,

140 dose/duration of Gn, number of oocytes retrieved, type of fertilization and DFP levels on the number

141 of blastocysts frozen and blastocyst formation rate, respectively. As shown in Table 3, the results

142 indicated that DFP was negatively associated with number of blastocysts frozen ( $\beta \pm$ SE: Group B vs

143 Group $\mathrm{A}=-0.319 \pm 0.115, \mathrm{P}=0.006$; Group $\mathrm{C}$ vs Group $\mathrm{A}=-0.432 \pm 0.154, \mathrm{P}=0.005$ ). As shown

144 in Table 4, the results indicated that DFP was also negatively associated with blastocyst formation

145 rate $(\beta \pm \mathrm{SE}$ : Group $\mathrm{B}$ vs Group $\mathrm{A}=-0.035 \pm 0.016, \mathrm{P}=0.031$; Group $\mathrm{C}$ vs Group $\mathrm{A}=-0.039 \pm$

$1460.021, \mathrm{P}=0.067)$.

147 Binary logistic regression analysis was carried out to evaluate the effect of type of infertility,

148 dose/duration of Gn, number of oocytes retrieved, type of fertilization and DFP levels on low

149 blastocysts frozen (Table 5). The results showed that compared with Group A, the OR for low 
$151 \mathrm{C}(95 \% \mathrm{CI}=1.014-1.979, \mathrm{P}=0.041)$.

\section{Discussion}

153 LH plays an essential physiological role in follicle steroidogenesis and development, as well as

154 oocyte maturation [20, 21]. Since the introduction of GnRH-a prolonged protocol, pituitary

155 desensitisation has usually been profound and endogenous LH level has been suppressed to be very

156 low $(<1.0 \mathrm{IU} / \mathrm{L})$. There is not any data available in published literature in regards to when to

157 administer HCG trigger in GnRH-a prolonged protocol. Some clinicians believe that the timing could

158 be determined using the same criteria as are used in short GnRH-a long protocol. However, the

159 traditional HCG trigger timing criteria are not strict, and the decision remains controversial [2 - 5].

160 The previous views were that follicular size was positively related to follicular maturity, fertilisation

161 and subsequent development $[1,2,5,12]$. Oocytes derived from large follicles (14 21 mm; mean

162 diameter: $19.1 \pm 2.1 \mathrm{~mm}$ ) seem to be more inclined to form high-quality embryos in $\mathrm{GnRH}$

163 antagonist protocol [13]. Therefore, in actual practice, the HCG trigger time is usually delayed [8].

164 On the contrary, a previous study reported that oocytes in oversized follicles in the same protocol

165 might decrease in quality, and the recovery of oocytes [5]. However, our results showed that the DFP

166 groups did not differ in terms of oocyte maturation rate or normal fertilisation rate. This was

167 consistent with the views of previous studies: enlarging follicle size might not improve oocyte

168 maturation or fertilisation [4, 14 - 16].

169 In our study, the clinical pregnancy rate and the implantation rate seemed to decrease as DFP

170 increased (Group B vs Group C). This could be explained by the fact that a larger DFP might be

171 negatively associated with satisfactory pregnancy outcomes. Consistent with our results, one study

172 showed that high-quality embryo rate, pregnancy rate, and implantation rate were significantly higher 
173 in the low proportion group (diameter $\geqslant 18 \mathrm{~mm}$ divided by the total number of follicles, low

174 proportion: <15\%; middle proportion: 15 - 27\%; high proportion: >27\% in short GnRH-a long

175 protocol) [8]. In another two randomised controlled trials of GnRH antagonist cycles, enlarging

176 follicle size by delaying HCG administration by one or two days after the time that three follicles had

177 reached $17 \mathrm{~mm}$, corresponded with a decrease in ongoing pregnancy rates in the delayed group [4,

178 14]. Availability of surplus embryos for freezing was lower when delaying two days compared with

179 delaying one day, but this did not reach statistical significance [14]. Interestingly in our study, as

180 DFP increased, the number of blastocysts frozen and blastocysts formation rate decreased

181 significantly. Multiple linear regression results showed that the number of blastocysts frozen and

182 blastocysts formation rate were negatively associated to DFP. Furthermore, increasing DFP is a risk

183 factor for low blastocysts frozen. These results revealed that the overgrowth of dominant follicles

184 might lead to oocyte post-maturity, which in turn could have an inverse impact on the quality of

185 oocytes and ultimately lead to unsatisfying pregnant outcomes $[8,18]$. This phenomenon may be

186 related to the increased incidence of ultrastructural abnormalities in the oocytes, for example, the

187 appearance of degenerate organelles-smooth surface endoplasmic reticulum (sER) [22]. Embryos that

188 accumulate the sER may have a low rate of blastocyst formation and poor pregnancy outcomes [23].

189 It is believed that sER aggregation is related to high E2 levels on HCG day and long-term Gn

190 stimulation [22]. From the results of clinical pregnancy and available blastocysts, it is speculated that

191 excessive delay of the trigger might negatively affect the cumulative pregnancy rate. But further

192 prospective study should be needed.

193 There are several advantages and limitations in our study. The most important innovation is the

194 discovery that the larger the DFP, the smaller the number of blastocysts frozen and blastocyst

195 formation rate. In other words, increasing DFP is a risk factor for low blastocysts frozen, and is also

196 negatively associated with good clinical outcomes in general. In addition, our study is the first to 
analyse the GnRH-a prolonged protocol in terms of HCG trigger time. Finally, for the analysis of the

198 DFP groupings and clinical outcomes, we used a large panel of data, established multiple linear

199 regression and binary logistic regression, and conducted a thorough and comprehensive evaluation of

200 these relationships. To a certain extent, it would provide useful information for clinical decision-

201 making. The limitations are as follows: First, due to its retrospective nature, some confounding bias

202 may exist. Second, we lack cumulative pregnancy rate results. It is hard to query and count the data

203 for such a large sample. In addition, we screened standardised young patients with normal ovarian

204 responses. The results may be not applicable to older patients or patients with low or high ovarian

205 responses.

206 In all, pursuing more and larger follicles may be of no benefit to clinical outcomes. This practice

207 might increase patient cost and time to a certain extent, without increasing the oocyte maturation rate,

208 normal fertilisation rate, or the number of embryos available for transfer. It might be preferable to

209 trigger as early as possible with patients who have not formed any available blastocysts (surprisingly,

210 these patients account for 1/5 calculated from our data), to avoid diminishing oocyte and embryo

211 quality, and avoid consequently poor IVF/ICSI outcomes.

\section{Conclusion}

213 Excessive delay of the HCG trigger during prolonged pituitary downregulation might reduce the

214 developmental potential of oocytes and reduce the number of available blastocysts, which might

215 result in a lower cumulative pregnancy rate. But further confirmation by strict prospective

216 randomized controlled study should be needed.

\section{Declarations}

\section{Ethics approval and consent to participate}


219 This study was approved by the Institutional Review Board at Huazhong University of Science and

220 Technology, Wuhan, China. All of the participants provided informed written consent.

\section{Consent for publication}

222 Not applicable.

\section{Availability of data and materials}

224 The datasets used and/or analysed during the current study are available from the corresponding 225 author upon reasonable request.

\section{Conflict of Interest}

227 The authors declare that the research was conducted in the absence of any commercial or financial 228 relationships that could be construed as a potential conflict of interest.

\section{Funding}

230 This work was supported by the National Key Research and Development Project [grant number

231 2018YFA0108401] and the National Natural Science Foundation [grant number 81571464] of China.

\section{Author Contributions}

233 K.Q. designed the study and revised the manuscript. HM.S. collected data, performed the statistical

234 analyses, and wrote manuscript. YH.L., J.L., TT.L., LC.J., and XY.H. collected the data. All authors 235 contributed to the interpretation of the results and editing of the manuscript.

\section{Acknowledgments}


237 We thank the staff at the Reproductive Medicine Center of Tongji Hospital for their outstanding 238 support.

239 References

240 1. Rosen MP, Shen S, Dobson AT, Rinaudo PF, McCulloch CE, Cedars MI: A quantitative

241 assessment of follicle size on oocyte developmental competence. Fertil Steril 2008, 90(3):684-690.

242 2. Lin HY, Li Y, Wang WJ, Qiu Q, Zhang QX, Li Y: Role of the proportion of dominant follicles in

243 patients with polycystic ovary syndrome undergoing in vitro fertilization-embryo transfer. Chin Med

244 J (Engl) 2019, 132(12):1448-1453.

245 3. Mochtar MH, Custers IM, Koks CA, Bernardus RE, Verhoeve HR, Mol BW, van Wely M, van der

246 Veen F: Timing oocyte collection in GnRH agonists down-regulated IVF and ICSI cycles: a

247 randomized clinical trial. Hum Reprod 2011, 26(5):1091-1096.

248 4. Kolibianakis EM, Albano C, Camus M, Tournaye H, Van Steirteghem AC, Devroey P:

249 Prolongation of the follicular phase in in vitro fertilization results in a lower ongoing pregnancy rate 250 in cycles stimulated with recombinant follicle-stimulating hormone and gonadotropin-releasing

251 hormone antagonists. Fertil Steril 2004, 82(1):102-107.

252 5. Hu X, Luo Y, Huang K, Li Y, Xu Y, Zhou C, Mai Q: New Perspectives on Criteria for the 253 Determination of HCG Trigger Timing in GnRH Antagonist Cycles. Medicine (Baltimore) 2016, 254 95(20):e3691.

255 6. Falagario M, Trerotoli P, Chincoli A, Cobuzzi I, Vacca MP, Falagario D, Nardelli C, Depalo R:

256 Dynamics of the development of multiple follicles by early versus late hCG administration in ART 257 program. Gynecol Endocrinol 2017, 33(2):105-108. 
7. European, Middle East Orgalutran Study G: Comparable clinical outcome using the GnRH antagonist ganirelix or a long protocol of the GnRH agonist triptorelin for the prevention of premature LH surges in women undergoing ovarian stimulation. Hum Reprod 2001, 16(4):644-651.

8. Li Y, Li RQ, Ou SB, Ren L, Zhang NF, Wei LN, Zhang QX, Yang DZ: Association between the proportion of dominant follicles and oocyte developmental competence. J Assist Reprod Genet 2014, 31(12):1599-1604.

9. Ectors FJ, Vanderzwalmen P, Van Hoeck J, Nijs M, Verhaegen G, Delvigne A, Schoysman R, Leroy F: Relationship of human follicular diameter with oocyte fertilization and development after in-vitro fertilization or intracytoplasmic sperm injection. Hum Reprod 1997, 12(9):2002-2005.

10. Haines CJ, Emes AL: The relationship between follicle diameter, fertilization rate, and microscopic embryo quality. Fertil Steril 1991, 55(1):205-207.

11. Bergh C, Broden H, Lundin K, Hamberger L: Comparison of fertilization, cleavage and pregnancy rates of oocytes from large and small follicles. Hum Reprod 1998, 13(7):1912-1915. follicular diameters and flushing versus no flushing on oocyte maturity, fertilization rate and embryo quality. J Assist Reprod Genet 2014, 31(1):73-77. competence of oocytes retrieved from the leading follicle in controlled ovarian stimulated cycles. Int J Fertil Steril 2013, 6(4):272-277. gonadotrophin (hCG) hormone administration in IVF protocols using GnRH antagonists: a randomized controlled trial. Hum Fertil (Camb) 2012, 15(3):134-139. 
preimplantation development in relation to follicle diameter in gonadotropin-releasing hormone agonist or antagonist treatments. Fertil Steril 2006, 85(3):578-583.

Association among diameter and volume of follicles, oocyte maturity, and competence in intracytoplasmic sperm injection cycles. Minerva Ginecol 2015, 67(5):397-403.

17. Venetis CA, Kolibianakis EM, Bosdou JK, Tarlatzis BC: Progesterone elevation and probability of pregnancy after IVF: a systematic review and meta-analysis of over 60000 cycles. Hum Reprod Update 2013, 19(5):433-457.

18. Wirleitner B, Okhowat J, Vistejnova L, Kralickova M, Karlikova M, Vanderzwalmen P, Ectors F, competence, blastocyst development and live-birth rate: optimal follicle size for oocyte retrieval. Ultrasound Obstet Gynecol 2018, 51(1):118-125. Analogue Suppression Protocols in IVF/ICSI Cycles in Patients of Various Age Ranges. PLoS One 2015, 10(7):e0133887. of endogenous luteinizing hormone serum levels on progesterone elevation on the day of human 298 chorionic gonadotropin administration. Fertil Steril 2011, 96(3):600-604. luteinizing hormone does not impair the outcome of human menopausal gonadotropin and medroxyprogesterone acetate treatment cycles. Sci Rep 2018, 8(1):13835. 
302 22. Otsuki J, Okada A, Morimoto K, Nagai Y, Kubo H: The relationship between pregnancy outcome

303 and smooth endoplasmic reticulum clusters in MII human oocytes. Hum Reprod 2004, 19(7):1591-

3041597.

305 23. Setti AS, Figueira RC, de Almeida Ferreira Braga DP, Azevedo MC, Iaconelli A, Jr., Borges E,

306 Jr.: Oocytes with smooth endoplasmic reticulum clusters originate blastocysts with impaired

307 implantation potential. Fertil Steril 2016, 106(7):1718-1724. 
Table 1. Baseline Characteristics and Clinical Outcomes.

\begin{tabular}{|c|c|c|c|c|}
\hline & Group A <30\% & Group B 30\%-60\% & Group $C \geqslant 60 \%$ & $\mathrm{P}$ \\
\hline No. of cycles & 398 & 1230 & 297 & - \\
\hline Duration of infertility(years) & $3.21 \pm 1.98$ & $3.35 \pm 2.14$ & $2.97 \pm 2.06$ & 0.026 \\
\hline Maternal age (years) & $29.17 \pm 2.94$ & $29.19 \pm 2.84$ & $29.13 \pm 2.60$ & 0.939 \\
\hline BMI $\left(\mathrm{kg} / \mathrm{m}^{2}\right)$ & $22.24 \pm 3.03$ & $22.19 \pm 3.09$ & $22.42 \pm 2.94$ & 0.500 \\
\hline $\mathrm{AFC}$ & $16.55 \pm 6.42$ & $16.39 \pm 6.15$ & $14.79 \pm 5.01 * *$ & $<0.001$ \\
\hline Basal FSH (mIU/ml) & $7.27 \pm 1.79$ & $7.23 \pm 1.69$ & $7.32 \pm 1.94$ & 0.685 \\
\hline $\mathrm{AMH}(\mathrm{ng} / \mathrm{ml})$ & $6.47 \pm 4.20$ & $6.59 \pm 4.00$ & $5.72 \pm 3.52 * *$ & 0.003 \\
\hline Dose of Gn (IU) & $2435.31 \pm 962.72$ & $2401.90 \pm 855.15$ & $2511.15 \pm 811.93$ & 0.149 \\
\hline Duration of Gn (days) & $11.31 \pm 2.01$ & $11.25 \pm 2.11$ & $10.99 \pm 1.84$ & 0.087 \\
\hline Dose/Duration of Gn (IU/d) & $214.10 \pm 70.19$ & $213.27 \pm 63.17$ & $228.88 \pm 64.52 * *$ & 0.001 \\
\hline E2 (HCG day, pg/ml) & $2322.80 \pm 1083.71$ & $2289.81 \pm 1047.77$ & $2283.90 \pm 1065.46$ & 0.849 \\
\hline P (HCG day, ng/ml) & $0.72 \pm 0.34$ & $0.79 \pm 0.52 *$ & $0.82 \pm 0.34 * *$ & 0.010 \\
\hline No. of $\geqslant 14 \mathrm{~mm}$ follicles & $11.92 \pm 3.27$ & $11.21 \pm 3.17 *$ & $9.45 \pm 2.95 * *$ & $<0.001$ \\
\hline No. of oocytes retrieved & $12.74 \pm 3.22$ & $12.22 \pm 3.35 *$ & $11.59 \pm 3.29 * *$ & $<0.001$ \\
\hline \multicolumn{5}{|l|}{ Oocyte maturation rate $(\%)$} \\
\hline IVF & $86.10 \pm 14.39$ & $88.17 \pm 14.22$ & $87.31 \pm 14.57$ & 0.101 \\
\hline ICSI & $82.27 \pm 14.87$ & $80.59 \pm 14.88$ & $81.41 \pm 13.05$ & 0.553 \\
\hline \multicolumn{5}{|l|}{ Normal fertilisation rate $(\%)$} \\
\hline IVF & $62.70 \pm 16.99$ & $63.26 \pm 18.53$ & $61.74 \pm 19.25$ & 0.525 \\
\hline ICSI & $57.53 \pm 19.46$ & $57.78 \pm 19.08$ & $55.16 \pm 17.80$ & 0.573 \\
\hline Endometrial thickness (mm) & $12.26 \pm 2.58$ & $12.30 \pm 2.58$ & $12.01 \pm 2.38$ & 0.223 \\
\hline No. of blastocysts frozen & $3.05 \pm 2.35$ & $2.60 \pm 2.16^{*}$ & $2.32 \pm 2.12 * *$ & $<0.001$ \\
\hline Blastocyst formation rate $(\%)$ & $42.38 \pm 26.59$ & $38.69 \pm 27.16^{*}$ & $38.26 \pm 28.27 * *$ & 0.047 \\
\hline
\end{tabular}


ET 1 embryo

Clinical pregnancy rate(\%) 58.20(188/323)

$65.30(510 / 781) \quad 62.82(98 / 156)$

0.084

ET 2 embryos

Clinical pregnancy rate(\%) 68.00(51/75)

$73.94(332 / 449)$

$67.38(95 / 141)$

0.232

Implantation rate $(\%)$

$48.67(73 / 150)$

$57.57(517 / 898)^{*}$

$50.71(143 / 282)$

0.031

$310 \mathrm{BMI}=$ body mass index $; \mathrm{AFC}=$ antral follicle counting $; \mathrm{FSH}=$ follicle stimulating hormone; IVF =

311 in vitro fertilization; ICSI = intracytoplasmic sperm injection.

$312 *$ Group B versus Group A, P < 0.05; ** Group C versus Group A, P < 0.05 . 
314 Table 2. Logistics Regression Analysis of Clinical Pregnancy.

\begin{tabular}{llll}
\hline & OR & $95 \%$ CI & P \\
\hline BMI & 0.935 & $0.904,0.966$ & $<0.001$ \\
Dose/Duration of Gn & 0.992 & $0.990,0.993$ & 0.001 \\
No. of oocytes retrieved & 0.950 & $0.920,0.980$ & \\
No. of embryo transfer & & & \\
2 & 1.469 & $1.180,1.8301$ \\
1 & REF & & \\
DFP & & & \\
Group A & REF & & 0.023 \\
Group B & 1.345 & $1.041,1.738$ & 0.216 \\
Group C & 1.242 & $0.881,1.751$ & \\
\hline
\end{tabular}

$315 \mathrm{REF}=$ reference value. $\mathrm{P}$-value of $<0.05$ indicated statistical significance. 
317 Table 3. Multiple linear regression analysis of number of blastocysts frozen.

\begin{tabular}{|c|c|c|c|c|}
\hline & $\beta \pm$ Standard error & Standardized $\beta$ & $\mathrm{t}$ & $\mathrm{P}$ \\
\hline \multicolumn{5}{|l|}{ Infertility } \\
\hline Secondary & $0.194 \pm 0.097$ & 0.042 & 2.010 & 0.045 \\
\hline Primary & REF & & & \\
\hline Dose/Duration of Gn & $-0.003 \pm 0.001$ & -0.078 & -3.692 & $<0.001$ \\
\hline No. of oocytes retrieved & $0.252 \pm 0.014$ & 0.380 & 18.051 & $<0.001$ \\
\hline \multicolumn{5}{|l|}{ Fertilization } \\
\hline ICSI & $-0.513 \pm 0.103$ & -0.104 & -4.966 & $<0.001$ \\
\hline IVF & $\mathrm{REF}$ & & & \\
\hline \multicolumn{5}{|l|}{ DFP } \\
\hline Group A & REF & & & \\
\hline Group B & $-0.319 \pm 0.115$ & -0.070 & -2.769 & 0.006 \\
\hline Group C & $-0.432 \pm 0.154$ & -0.071 & -2.798 & 0.005 \\
\hline
\end{tabular}

$318 \mathrm{REF}=$ reference value. $\mathrm{P}$-value of $<0.05$ indicated statistical significance. 
320 Table 4. Multiple linear regression analysis of blastocyst formation rate.

\begin{tabular}{|c|c|c|c|c|}
\hline & $\beta \pm$ Standard error & Standardized $\beta$ & $\mathrm{t}$ & $\mathrm{P}$ \\
\hline \multicolumn{5}{|l|}{ Infertility } \\
\hline Secondary & $0.027 \pm 0.012$ & 0.055 & 2.330 & 0.020 \\
\hline Primary & REF & & & \\
\hline Dose/Duration of Gn & $-0.0004 \pm 0.0001$ & -0.095 & -4.038 & $<0.001$ \\
\hline No. of oocytes retrieved & $0.006 \pm 0.002$ & 0.071 & 3.029 & 0.002 \\
\hline \multicolumn{5}{|l|}{ Fertilization } \\
\hline ICSI & $-0.027 \pm 0.014$ & -0.045 & -1.914 & 0.056 \\
\hline IVF & REF & & & \\
\hline \multicolumn{5}{|l|}{ DFP } \\
\hline Group A & $\mathrm{REF}$ & & & \\
\hline Group B & $-0.035 \pm 0.016$ & -0.061 & -2.160 & 0.031 \\
\hline Group C & $-0.039 \pm 0.021$ & -0.052 & -1.833 & 0.067 \\
\hline
\end{tabular}

$321 \mathrm{REF}=$ reference value. $\mathrm{P}$-value of $<0.05$ indicated statistical significance. 
323 Table 5. Logistics regression analysis of low blastocysts frozen $(\leqslant 1)$.

\begin{tabular}{llll}
\hline & OR & $95 \%$ CI & P value \\
\hline Infertility & & & \\
Secondary & 0.768 & $0.621,0.949$ & \\
Primary & REF & & $<0.001$ \\
Dose/Duration of Gn & 1.003 & $1.001,1.005$ & $<0.001$ \\
No. of oocytes retrieved & 0.832 & $0.806,0.858$ & 0.001 \\
Fertilization & & & \\
ICSI & 1.464 & $1.175,1.826$ & \\
IVF & REF & & \\
DFP & & & \\
Group A & REF & $1.014,1.698$ & 0.039 \\
Group B & 1.312 & $1.014,1.979$ & \\
Group C & 1.417 & & \\
\hline
\end{tabular}

324 Low Blatocysts Frozen = number of blastocysts frozen $\leqslant 1 ; \mathrm{REF}=$ reference value. $\mathrm{P}$-value of $<$

3250.05 indicated statistical significance. 